\title{
BMJ Open Prospective open-label non-inferiority randomised controlled trial comparing letrozole and mifepristone pretreatment in medical management of first trimester missed miscarriage: study protocol
}

\author{
Libei Du (1) , ${ }^{1}$ Raymond Hang Wun Li (10) ,1,2 Kristina Gemzell-Danielsson, ${ }^{1,2,3}$ \\ Yan Hong Du, ${ }^{1}$ Li Zhang, ${ }^{1}$ Wei Yu Diao, ${ }^{1}$ Pak Chung Ho ${ }^{1,2}$
}

To cite: Du L, Li RHW, GemzellDanielsson K, et al. Prospective open-label non-inferiority randomised controlled trial comparing letrozole and mifepristone pretreatment in medical management of first trimester missed miscarriage: study protocol. BMJ Open 2022;12:e052192. doi:10.1136/ bmjopen-2021-052192

- Prepublication history for this paper is available online. To view these files, please visit the journal online (http://dx.doi. org/10.1136/bmjopen-2021 052192).

Received 11 April 2021 Accepted 06 December 2021

Check for updates

(C) Author(s) (or their employer(s)) 2022. Re-use permitted under CC BY-NC. No commercial re-use. See rights and permissions. Published by BMJ.

1Department of Obstetrics and Gynecology, University of Hong Kong-Shenzhen Hospital, Shenzhen, China

${ }^{2}$ Department of Obstetrics and Gynaecology, The University of Hong Kong, Queen Mary Hospital, Pokfulam, Hong Kong ${ }^{3}$ Department of Women's and Children's Health, Karolinska Institutet, Karolinska University Hospital, Stockholm, Sweden

Correspondence to Dr Raymond Hang Wun Li; raymondli@hku.hk

\section{ABSTRACT}

Introduction Medical treatment is a less invasive alternative to surgical management of missed miscarriage. Studies have shown that pretreatment with mifepristone can increase the complete abortion rate in management of first-trimester missed miscarriage compared with misoprostol alone. Two studies have also shown that pretreatment with letrozole could increase the efficacy compared with misoprostol alone. So far, there is no trial comparing letrozole and mifepristone pretreatment for missed miscarriage. We designed this randomised controlled trial to test the hypothesis that for first-trimester missed miscarriage, letrozole pretreatment is non-inferior to mifepristone pretreatment followed by misoprostol in terms of complete abortion rate.

Methods and analysis This is a prospective open-label non-inferiority randomised controlled trial conducted in a single centre. In total, 294 women diagnosed with firsttrimester missed miscarriage opting for medical treatment is recruited with informed consent. They are randomly assigned to receive mifepristone or letrozole pretreatment. In the mifepristone group, each woman takes $200 \mathrm{mg}$ mifepristone orally followed $24-48$ hours later by $800 \mu \mathrm{g}$ misoprostol vaginally. In the letrozole group, each woman takes $10 \mathrm{mg}$ letrozole orally per day for 3 days, followed by $800 \mu \mathrm{g}$ misoprostol vaginally on the third day of letrozole administration. Follow-up is conducted on days 15 and 42 after misoprostol administration. The primary outcome is the overall complete abortion rate. Secondary outcomes include side effects and complications during the study period. Data will be analysed with both intention-to-treat and per protocol approaches. A $p<0.05$ will be considered as indicating statistical significance.

Ethics and dissemination Ethics approval has been obtained from the Institutional Review Board of the University of Hong Kong-Shenzhen Hospital with approval number: (2020)166. Findings will be disseminated in a peer-reviewed journal and in national and/or international meetings to guide future practice.

Trial registration number ChiCTR2000041480.

\section{INTRODUCTION}

Miscarriage is a common early pregnancy complication that occurs in $15 \%-20 \%$ of

\section{Strengths and limitations of this study}

- This study is the first randomised control trial to provide robust evidence comparing the two regimens studied for medical treatment of first-trimester missed miscarriage.

- The participants and the nurses administering the treatment drugs are not blinded to the group assignment due to practical reasons, although the clinicians and nurses conducting the follow-up assessment are blinded.

- It is anticipated that some participants will default the follow-up at day 42, although a drop-out rate of $10 \%$ has already been accounted for in the sample size calculation.

all clinical pregnancies, and the majority of miscarriages occur in the first trimester. ${ }^{12}$ Missed miscarriage and incomplete miscarriage are two main types of miscarriages that require medical intervention. Missed miscarriage is diagnosed when a non-viable pregnancy or anembryonic pregnancy is identified on ultrasound scan without associated pain or bleeding, which is also known as delayed or silent miscarriage or missed abortion. ${ }^{3}$ Incomplete miscarriage is diagnosed when pregnancy tissue has been partly expelled by the uterus. ${ }^{4}$ The treatment options for early pregnancy loss include expectant management, medical management and surgical evacuation. ${ }^{5}$ Surgical evacuation is a quick procedure with high success rate, but it is associated with risks of mechanical injury and postoperative intrauterine adhesions which may affect future fertility. ${ }^{6}$ Medical treatment of miscarriage is safe and less costly than surgical evacuation, and can avoid the surgical complications. Disadvantages of medical management include longer 
duration of bleeding and higher risk of unplanned surgical procedures such as curettage. ${ }^{78}$ Compared with expectant management, it shortens the time-to-abortion in a non-invasive manner, which may lessen the psychological burden to some women. ${ }^{6-11}$

Misoprostol induces uterine myometrial contractions as well as cervical softening and dilation. It is widely used to induce abortion either alone or following pretreatment with mifepristone. Vaginal misoprostol was recommended for medical treatment of missed miscarriage by the clinical guideline of the National Institute for Health and Care Excellence (NICE), UK. ${ }^{4}$ Treatment with repeat doses of misoprostol is effective, safe and acceptable. When used alone for medical management of miscarriage, the standard dose of misoprostol is $800 \mu \mathrm{g}$ vaginally or $600 \mu \mathrm{g}$ sublingually. ${ }^{12}$ It may work less well in women with a closed cervix. Up to $15 \%-40 \%$ of women with missed miscarriage need a second dose of misoprostol or eventually need surgical evacuation due to failed or incomplete abortion. ${ }^{12-16}$ Mifepristone pretreatment has been shown to increase the success rate. The reported complete abortion rate of pretreatment with mifepristone followed by misoprostol for the medical management of first trimester missed miscarriage in previous clinical trials has ranged from $79 \%$ to $87 \%$, and that of misoprostol alone ranged from $58 \%$ to $76 \% .{ }^{17-23}$ As access to mifepristone is restricted in some countries, and some patients may have contraindications to the use of mifepristone, it is important to find a replacement for mifepristone for medical treatment of missed miscarriage. ${ }^{24}$ We propose that letrozole may be a good alternative.

Letrozole is an oral aromatase inhibitor, ${ }^{25}$ which can inhibit oestrogen synthesis. It has been shown to be useful when combined with misoprostol in medical abortion as a possible alternative to mifepristone. ${ }^{26}$ A pilot study showed that a combination of letrozole and misoprostol used for termination of pregnancy up to 63 days achieved a complete abortion rate of $95 \% .{ }^{27}$ Another pilot study showed that combination of letrozole, mifepristone and misoprostol used for terminations of pregnancy up to 63 days achieved a complete abortion rate of $98 \% .^{28} \mathrm{~A}$ randomised controlled trial (RCT) has reported that pretreatment with letrozole followed by misoprostol can improve the complete abortion rate and reduce the interval between induction and abortion for first trimester miscarriage. This study showed a complete abortion rate of $78 \%$ in the letrozole pretreatment group and $39 \%$ in the placebo group. The interval between induction and abortion in the letrozole group was 1.42 days which was shorter than that in the placebo group (3.09 days). ${ }^{29}$ Another RCT showed a complete abortion rate of $93.7 \%$ in the letrozole plus misoprostol group compared with $68.7 \%$ in the misoprostol alone group for the treatment of first trimester non-viable pregnancies. ${ }^{30}$

The above randomised controlled studies suggested that pretreatment with either mifepristone or letrozole followed by misoprostol are both effective in the management of first-trimester missed miscarriage. However, so far, there is no trial comparing the effectiveness of letrozole versus mifepristone as pretreatment in this scenario.

We hypothesise that medical treatment for missed miscarriage with letrozole pretreatment is non-inferior to mifepristone pretreatment followed by misoprostol. The findings of this study will guide the development of an alternative regimen for medical treatment of missed miscarriage.

\section{MATERIALS AND METHODS \\ Study design and setting}

This non-inferiority, open-label randomised trial is conducted at the Department of Obstetrics and Gynaecology, The University of Hong Kong-Shenzhen Hospital, Shenzhen, China. Eligible women requesting medical management of missed miscarriage who are willing to participate are recruited.

\section{Sample size estimation}

According to the literature, complete abortion rate with mifepristone and misoprostol is about $84 \% .{ }^{20}$ Adopting a non-inferiority margin of $6 \%$, a minimum sample size of 132 per group (in the ratio of 1:1) will be needed to achieve power of $80 \%$ and type I error of $5 \%$. Accounting for a drop-out rate of about $10 \%$, a sample size of 147 in each arm, that is, 294 women in total will be recruited. We have first conducted a pilot analysis on 20 patients from each group, which revealed that the complete abortion rate in both groups were $95 \%$ without statistically significant difference; hence no change in the sample size is needed.

\section{Randomisation}

Randomisation is performed when the women attend the out-patient clinic because of missed miscarriage and are about to commence medical management. Eligible women are randomly assigned to either the mifepristone or letrozole pretreatment group. The randomisation table is generated from www.sealedenvelope.com in blocks of 10 . The vouchers showing the assigned arms according to the randomisation list are sealed in opaque envelopes which are opened on recruitment of each patient in sequence. Medication is administered to the patient according to the randomisation voucher by a nurse who is not involved in group allocation of the women.

\section{Selection of subjects}

Inclusion criteria include: (1) women aged 18 years or above who are diagnosed with first trimester missed miscarriage at 5-12 complete weeks of gestation, (2) having singleton pregnancy, (3) agreeing to participate in the trial voluntarily and (4) having no contraindications to medical abortion.

The diagnosis of missed miscarriage is made both by ultrasound scan and clinical examination. Speculum examination is performed to rule out incomplete or inevitable miscarriage. The following findings on transvaginal 
ultrasound suggests the diagnosis of missed miscarriage: crown-rump length of $7 \mathrm{~mm}$ or greater with no heartbeat, or mean sac diameter of $25 \mathrm{~mm}$ or greater with no embryo visualised in repeated ultrasound scans by two sonographers or on two occasions at least 7 days apart; absence of embryo with heartbeat 2 weeks or more after a scan that showed a gestational sac without a yolk sac; absence of embryo with heartbeat 11 days or more after a scan that showed a gestational sac with a yolk sac. ${ }^{34}$

Exclusion criteria include: (1) incomplete or inevitable miscarriage (defined by the clinical finding of an open cervix and bleeding), (2) suspected ectopic pregnancy, (3) history of heart, liver, kidney disease or adrenal insufficiency, (4) abnormal uterine lesions such as adenomyosis, fibroids or congenital malformations or intrauterine adhesion, (4) pregnancy with an intrauterine contraceptive device in situ, (5) history of coagulatory dysfunction or intake of anticoagulant drugs and (6) having haemoglobin level of less than $95 \mathrm{~g} / \mathrm{L}$. Informed written consent is obtained from all participants. The women can withdraw from the study for any reason at any time, and will receive the standard medical care in such case.

\section{Treatment procedures}

After obtaining written informed consent, randomisation is carried out as described above. Participants in the mifepristone group receive a single oral dose of $200 \mathrm{mg}$ mifepristone at home, followed $24-48$ hours later by $800 \mu \mathrm{g}$ misoprostol administered vaginally by the nurse or the woman herself in the hospital. Based on the regimen used in a previous study on medical termination of pregnancy, patients in the letrozole group receive oral letrozole at home, $10 \mathrm{mg}$ per day for three consecutive days, and on the morning of the third day they receive $800 \mu \mathrm{g}$ of misoprostol administered vaginally in the hospital. The participants are not blinded to their allocated treatment.

After misoprostol administration, the participants shall stay in the hospital for at least 3-6hours to observe for bleeding, tissue passage and severe adverse effects. A standard data form is used to record baseline demographics, medical history and obstetric history, timing of drug administration, side effects, timing of tissue expulsion, severity of abdominal pain and vaginal bleeding. Other side effects such as nausea, vomiting, diarrhoea, headache and fever are also recorded. Acceptability of the treatment method is assessed with the question 'Will you recommend this method of treatment to someone who need the treatment?'. We are not routinely checking the rhesus status nor providing anti-D rhesus prophylaxis to women undergoing medical treatment for miscarriage. Those receiving surgical treatment who are rhesus negative will receive $250 \mathrm{IU}$ anti-D immunoglobulin according to the latest NICE guideline. ${ }^{4}$ After discharge, the women are asked to record the duration of vaginal bleeding and occurrence of adverse events in the daily record charts until 42 days after misoprostol administration. The questionnaires are completed by the patients under guidance by the nurse, who are blinded to the allocation of the participants.

Participants who have no tissue passage at discharge from the hospital shall return for assessment 7 days after misoprostol use. Passage of gestational tissue, ongoing heavy vaginal bleeding and clinical signs of infection are noted. The clinician conducting the follow-up assessment is blinded to the group assigned. Women in whom the gestational sac has not been expelled by day 7 can choose to receive a second dose of $800 \mu \mathrm{g}$ misoprostol vaginally or $400 \mu \mathrm{g}$ misoprostol sublingually, to have expectant treatment or to undergo suction evacuation. Those who choose to have a second dose of misoprostol or expectant treatment are reviewed in another week. If the gestational sac is still not expelled 1 week after the second dose of misoprostol, suction evacuation shall be performed. All participants receive a telephone follow-up around 15 days and attend an in-person follow-up visit at 42 days after misoprostol administration to collect information about additional treatments or adverse events. In the mean time, the woman can return to the hospital if there is heavy vaginal bleeding, fever or severe abdominal pain. A pregnancy test is performed at the day 42 visit. If the result is negative, no further action is needed. If the result is positive or there is ongoing bleeding, an ultrasound scan is performed to guide further management. Complete abortion is considered if there is no gestational sac or fetal structure. Incomplete abortion is defined by a positive pregnancy test result and/or ongoing vaginal bleeding, together with sonographic finding of intrauterine material with a thickness of more than $15 \mathrm{~mm}$, and either expectant or surgical treatment may be offered as per informed decision of the patient.

The study pathway is illustrated in figure 1 .

\section{Indications for suction evacuation}

The indications for suction evacuation include persistent presence of a pregnancy sac 7-15days after the first or second misoprostol administration, heavy vaginal bleeding, or incomplete abortion after 42 days following misoprostol administration. This will be an informed decision by the patient after explanation about the alternative options of expectant and repeat medical management.

\section{Outcome measures}

The primary outcome is the rate of complete abortion, defined as the complete expulsion of the gestational product without the need for suction evacuation or other intervention till follow-up at 42 days. Secondary outcomes include the rate of side effects such as nausea, vomiting, fever, severe pain, severe bleeding and other complications during the study period, as well as patient satisfaction. The need for urgent surgical intervention will also be reported.

\section{Data analysis}

Data will be analysed with both the intention-to-treat and per protocol approaches. Categorical and continuous 


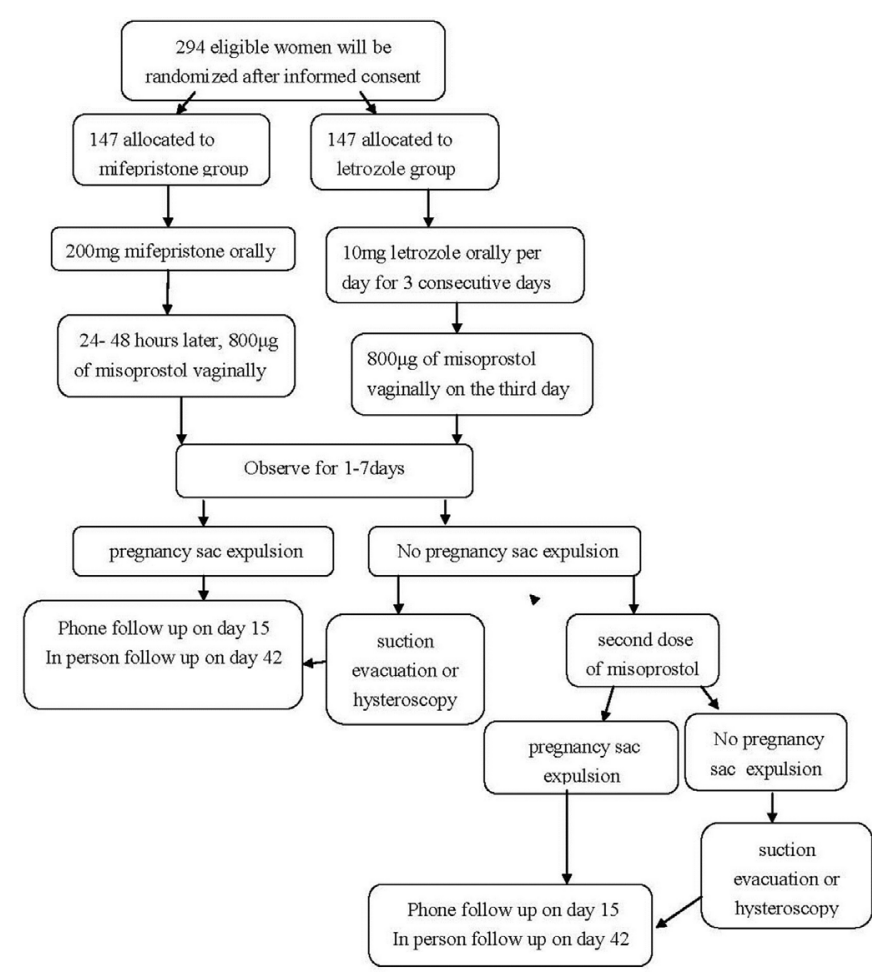

Figure 1 Flow chart of study pathway. If the pregnancy sac is not expulsed by day 7 , a second dose of misoprostol will be administered or surgical evacuation will be performed on day 8 as per patient's informed choice.

variables will be compared between groups by $\chi^{2}$ test, Student's t-test or Mann-Whitney U test as appropriate. $P$ values of less than 0.05 will be considered as indicating statistical significance. IBM SPSS Statistics V.25 software will be used for analysing the data. The investigator performing data analysis shall be blinded to the coding of the treatment groups.

\section{Assessment of safety}

Mifepristone and misoprostol are both in wide use and are the medications recommended by the WHO for medical abortion with well documented safety profile. Letrozole has been approved by the US Food and Drug Administration with well documented safety profile.

\section{Patient and public involvement}

There is no patient and public involvement in the design and execution of this study.

\section{Trial status}

Study recruitment has been started on 28 December 2020 , with 132 subjects having been recruited at the time of final revision of this manuscript.

\section{Financing and insurance}

The study is supported by internal departmental funding.

Acknowledgements We would like to express our thanks to other doctors and nurses in the Department of Obstetrics and Gynecology who participated in patient recruitment and follow-up for this study. We would also like to thank all patients who participated in the study.
Contributors LD, RHWL, KG-D and PCH took part in designing and developing the study protocol. LZ, YHD and WYD provided comments and advice on the study methodology and logistics. LD drafted the manuscript, with critical input from RHWL, KG-D and PCH. The manuscript was read and approved by all authors.

Funding The study is supported by internal departmental funding. Competing interests None declared.

Patient and public involvement Patients and/or the public were not involved in the design, or conduct, or reporting, or dissemination plans of this research.

Patient consent for publication Not applicable.

Provenance and peer review Not commissioned; externally peer reviewed.

Open access This is an open access article distributed in accordance with the Creative Commons Attribution Non Commercial (CC BY-NC 4.0) license, which permits others to distribute, remix, adapt, build upon this work non-commercially, and license their derivative works on different terms, provided the original work is properly cited, appropriate credit is given, any changes made indicated, and the use is non-commercial. See: http://creativecommons.org/licenses/by-nc/4.0/.

ORCID iDs

Libei Du http://orcid.org/0000-0003-3541-5006

Raymond Hang Wun Li http://orcid.org/0000-0002-7957-7798

\section{REFERENCES}

1 Regan L, Rai R. Epidemiology and the medical causes of miscarriage. Best Pract Res Clin Obstet Gynaecol 2000;14:839-54.

2 Stephenson MD. Recurrent early pregnancy loss. Semin Reprod Med 2011;29:461-2.

3 American College of Obstetricians and Gynecologists' Committee on Practice Bulletins-Gynecology. ACOG practice Bulletin No. 200: early pregnancy loss. Obstet Gynecol 2018;132:e197-207.

4 National Institute for Health and Clinical Excellence. NICE Guideline, no. 126. ectopic pregnancy and miscarriage: diagnosis and initial management. April 17, 2019. Available: https://www.nice.org.uk/ guidance/ng126 [Accessed 7 Aug 2020].

5 Jurkovic D, Overton C, Bender-Atik R. Diagnosis and management of first trimester miscarriage. BMJ 2013;346:f3676:1-7.

6 Sotiriadis A, Makrydimas G, Papatheodorou S, et al. Expectant, medical, or surgical management of first-trimester miscarriage: a meta-analysis. Obstet Gynecol 2005;105:1104-13.

7 Şahin HG, Şahin HA, Kocer M. Randomized outpatient clinical trial of medical evacuation and surgical curettage in incomplete miscarriage. Eur J Contracept Reprod Health Care 2001;6:141-4.

8 Society of Family Planning. Clinical guidelines: medical management of first-trimester abortion. Contraception 2014;89:148-61.

9 Graziosi GCM, Mol BW, Ankum WM, et al. Management of early pregnancy loss. Int J Gynaecol Obstet 2004;86:337-46.

10 Bagratee JS, Khullar V, Regan L, et al. A randomized controlled trial comparing medical and expectant management of first trimester miscarriage. Hum Reprod 2004;19:266-71.

11 Ngai SW, Chan YM, Tang OS, et al. Vaginal misoprostol as medical treatment for first trimester spontaneous miscarriage. Hum Reprod 2001:16:1493-6.

12 Wu H-L, Marwah S, Wang P, et al. Misoprostol for medical treatment of missed abortion: a systematic review and network meta-analysis. Sci Rep 2017;7:2161-70.

13 Neilson JP, Hickey M, Vazquez J. Medical treatment for early fetal death (less than 24 weeks). Cochrane Database Syst Rev 2006;3:CD002253.

14 Chen BA, Creinin MD. Contemporary management of early pregnancy failure. Clin Obstet Gynecol 2007:50:67-88.

15 Robledo C, Zhang J, Troendle J, et al. Clinical indicators for success of misoprostol treatment after early pregnancy failure. Int J Gynaecol Obstet 2007;99:46-51.

16 Creinin MD, Huang X, Westhoff C, et al. Factors related to successful misoprostol treatment for early pregnancy failure. Obstet Gynecol 2006;107:901-7.

17 Zhang J, Gilles JM, Barnhart K, et al. A comparison of medical management with misoprostol and surgical management for early pregnancy failure. N Engl J Med 2005;353:761-9.

18 Stockheim D, Machtinger R, Wiser A, et al. A randomized prospective study of misoprostol or mifepristone followed by misoprostol when needed for the treatment of women with early pregnancy failure. Fertil Steril 2006;86:956-60.

19 Grønlund A, Grønlund L, Clevin L, et al. Management of missed abortion: comparison of medical treatment with either mifepristone 
+ misoprostol or misoprostol alone with surgical evacuation. A multicenter trial in Copenhagen County, Denmark. Acta Obstet Gynecol Scand 2002;81:1060-5.

20 Schreiber CA, Creinin MD, Atrio J, et al. Mifepristone pretreatment for the medical management of early pregnancy loss. N Engl J Med 2018;378:2161-70.

21 Sinha P, Suneja A, Guleria K, et al. Comparison of mifepristone followed by misoprostol with misoprostol alone for treatment of early pregnancy failure: a randomized double-blind placebo-controlled trial. J Obstet Gynaecol India 2018;68:39-44.

22 Chu JJ, Devall AJ, Beeson LE, et al. Mifepristone and misoprostol versus misoprostol alone for the management of missed miscarriage (MifeMiso): a randomised, double-blind, placebo-controlled trial. Lancet 2020;396:770-8.

23 Hamel C, Coppus S, van den Berg J, et al. Mifepristone followed by misoprostol compared with placebo followed by misoprosto as medical treatment for early pregnancy loss (the triple $\mathrm{M}$ trial): a double-blind placebo-controlled randomised trial. EClinicalMedicine 2021;32:100716.

24 Dalenda C, Ines N, Fathia B, et al. Two medical abortion regimens for late first-trimester termination of pregnancy: a prospective randomized trial. Contraception 2010;81:323-7.
25 Malloch L, Rhoton-Vlasak A. An assessment of current clinical attitudes toward letrozole use in reproductive endocrinology practices. Fertil Steril 2013;100:1740-4.

26 Lee VCY, Ng EHY, Yeung WSB, et al. Misoprostol with or without letrozole pretreatment for termination of pregnancy: a randomized controlled trial. Obstet Gynecol 2011;117:317-23.

27 Yeung TWY, Lee VCY, Ng EHY, et al. A pilot study on the use of a 7-day course of letrozole followed by misoprostol for the termination of early pregnancy up to 63 days. Contraception 2012;86:763-9.

28 Chai J, Ho P-C. A pilot study on the combined use of letrozole, mifepristone and misoprostol in termination of first trimester pregnancy up to 9 weeks' gestation. Eur J Obstet Gynecol Reprod Biol 2013;171:291-4.

29 Torky HA, Marie H, ElDesouky E, et al. Letrozole vs. placebo pretreatment in the medical management of first trimester missed miscarriage: a randomized controlled trial. Geburtshilfe Frauenheilkd 2018;78:63-9.

30 Abbasalizadeh F, Sahhaf F, Sadeghi-Shabestari P, et al. Comparison between effect of letrozole plus misoprostol and misoprostol alone in terminating non-viable first trimester pregnancies: a single blind randomized trial. J Family Reprod Health 2018;12:27-33. 